\section{DNA typing of museum birds}

SIR - The recent report by Hagelberg et $a l .{ }^{1}$ of identification of human skeletal remains by DNA typing illustrates the synergistic effects derived from a combined use of the polymerase chain reaction (PCR) and microsatellite markers. While PCR greatly facilitates studies of ancient DNA (see Sykes's recent News and Views article ${ }^{2}$ ), microsatellites are highly feasible as genetic markers because of their abundancy and hypervariability ${ }^{3}$.

An interesting possibility in this context is to DNA-type museum specimens with the aid of PCR-analysed microsatellites. This would allow detailed genetic analyses of extinct species ${ }^{4}$ as well as population-genetic comparisons of present and past populations ${ }^{2}$. I have evaluated feathers as genomic DNA source for avian microsatellite analysis of museum specimens. Using microsatellite sequences isolated from the swallow (Hirundo rustica) and the pied flycatcher (Ficedula hypoleuca), I have typed birds of these species which are more than 100 years old.

DNA was prepared from the root part of single feather shafts (remiges and restrices provided by $\mathrm{G}$. Frisk and $\mathrm{S}$. Mathiasson, Swedish and Gothenburg Museums of Natural History) by a simple protease/boiling procedure in the presence of a chelating resin. Approximately $5 \%$ of a feather preparation was subjected to PCR, yielding autoradiography signals corresponding to $10-50 \mathrm{ng}$ template DNA. None of the primer pairs

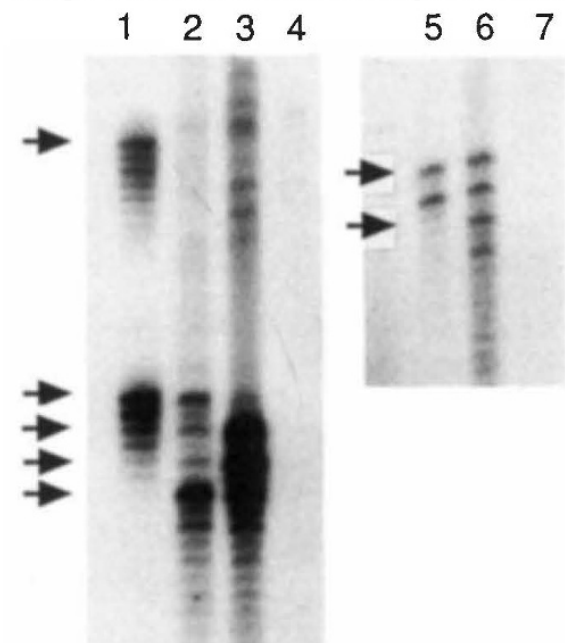

Examples of PCR amplifications of microsatellites from museum specimens of birds, separated by polyacrylamide gel electrophoresis. Lanes 1-4, amplifications from the pied flycatcher locus PTC3; lanes 5-7, amplifications from the swallow locus STG4. The birds were collected in 1924 (lane 2), 1926 (lane 3), 1890 (lane 5) and 1915 (lane 6). Lane 1 is a bird sampled in 1991. lanes 4 and 7 are negative controls for each locus, respectively. Alleles are arrowed. amplifies modern human DNA so it is unlikely that the PCR products arise from human contamination.

An analysis of five pied flycatchers collected in Sweden between 1912 and 1926 reveals that all the birds were heterozygous, together showing five different 130-140-base-pair (bp) alleles at PTC3, a thymine-cytosine dinucleotide repeat. Three swallows collected between 1860 and 1930 showed three different alleles at STG1 and STG4, two thymine-guanine dinucleotide repeats (about 210 and $140 \mathrm{bp}$, respectively). All alleles of the museum specimens are present in extant populations in Sweden.

Most previous studies of ancient DNA have focused on multicopy mitochondrial DNA sequences. Although genomic microsatellites have been successfully amplified from skeletal remains ${ }^{1}$, Hagelberg et al. warned of the low yields of nuclear DNA obtainable in bone preparations. Because old feathers seem to be
SIR - Lorenz ${ }^{1}$ has reported numerical experiments that cast doubt on the validity of estimates of the correlation dimension derived from experimental data. His warnings about the effect of strong and weak coupling should be heeded. But one of the features of his model, symmetry, may be partially responsible for some of the difficulties observed. Because many parameters have been set equal in his numerical experiments, the equations are symmetric under certain permutations of the variables. Indeed, the symmetry group is abstractly isomorphic to that of a square ( $4 \mathrm{~m}$ in crystallographic notation).

The theoretical basis of all 'delay coordinate' reconstruction methods is the embedding theorem of Takens ${ }^{2}$, which states that for 'generic' dynamical systems and observations, a suitable timedelay map is a topological embedding of phase space. However, symmetric systems are nongeneric, and violate the hypotheses of the Takens embedding theorem. For example, for reconstruction to work properly, certain eigenvalues must be simple -- symmetry, however, can force them to be multiple. There is thus no theoretical basis for applying traditional methods of phase space reconstruction to symmetric systems. The reconstruction map may not only fail to embed phase space: it may even fail to immerse it (embed with self-intersections) and thus create singularities. These in turn can produce numerical instability, and invalidate standard procedures that require local linear approximations (such as box- a good source for nuclear DNA, birds may be particularly well suited for DNA typing of museum specimens. The regular presence of large skin collections facilitates such approach.

A potential problem when typing old microsatellites may be numerous artefact bands generated through jumping PCR if DNA is degraded (ref. 5; S. Pääbo, personal communication). By comparing the ancient amplification products and the extra band-pattern obtained with modern DNA, discrepancies can be monitored.

\section{Department of Animal Breeding and}

Genetics,

Swedish University of Agricultural

Sciences,

Box 7055, S-750 07 Uppsala, Sweden

1. Hagelberg, E., Gray, I. C. \& Jeffreys, A. J. Nature 352. 427-429 (1991).

2. Sykes, B. Nature 352, 381-382 (1991)

3. Weber, J. L. \& May, P. E. Am. J. hum. Genet. 44 , 388-396 (1989).

4. Thomas, R. H. et al. Nature 340, 465-467 (1989)

5. Pääbo, S., Irwin, D. M. \& Wilson, A. C. J. biol. Chem 265, 4718-4721 (1990).

\title{
Symmetry and chaotic data
}

counts for dimensions). Instead, a symmetrically related set of observations must be used ${ }^{3,4}$. Moreover, they must take values in a representation $V$ of the symmetry group that is sufficiently complicated: the phase space must be subordinate to $V$ in the sense of Wassermann ${ }^{5}$.

Lorenz contrasts dimension estimates and reconstructions performed using two distinct variables, $Z_{7}$ and $Z^{*}$. The variable $Z_{7}$ is asymmetric, and so is the attractor in Lorenz's Fig. 4a. However, $Z^{*}$ is invariant under the full symmetry group, and phase space is not subordinate to the corresponding representation. It is not surprising that markedly different results are obtained.

It is therefore important to disentangle symmetry effects from those of weak versus strong coupling. This might be done by varying the coupling constants in Lorenz's model to destroy the symmetry, and repeating the analysis. When doing so it should be borne in mind that approximate symmetry will in practice lead to similar problems to those created by exact symmetry.

Mathematics Institute,

University of Warwick,

Coventry CV4 7AL, UK

1. Lorenz, E. Nature 353, 241 (1991).

2. Takens, F. in Dynamical Systems and Turbulence Warwick 1980 (eds Rand, D. A. \& Young, L.-S.) 366 (Springer, New York, 1981).

3. King, G. P. \& Stewart, I. N. in Nonlinear Equations in the Applied Sciences (eds Ames, W. F. \& Rogers, C. F.) 257 (Academic, Boston, 1991)

4. King, G. P. \& Stewart, I. N. Phase Space Reconstruction for Symmetric Dynamical Systems Preprint (Math. inst. Univ. Warwick, 1991)

5. Wassermann. A. Topology 8, 127 (1969). 